\title{
Analysis on solder ball shear testing conditions with a simple computational model
}

\author{
S.W. Ricky Lee \\ Electronic Packaging Laboratory, Department of Mechanical Engineering, \\ Hong Kong University Science \& Technology, Kowloon, Hong Kong \\ Xingjia Huang \\ Electronic Packaging Laboratory, Department of Mechanical Engineering, \\ Hong Kong University Science \& Technology, Kowloon, Hong Kong
}

\section{Keywords}

Plastic ball grid array, Solder joints,

Finite element analysis, Reliability

\section{Abstract}

This paper introduces a simple computational model for the analysis on the solder ball shear testing conditions. Both twodimensional (2-D) and threedimensional (3-D) finite element models are used to investigate the effect of shear ram speed on the solder ball shear strength of plastic ball grid array (PBGA) packages. An effective thickness is identified for the 2-D finite element analysis. By using this effective thickness as a scale factor, it is shown that the $2 \mathrm{D}$ model is feasible for the study of 3-D problems. The computational model is validated by experimental data in terms of load-displacement curves. The results from both testing and modeling indicate that the shear ram speed has a substantial effect on the solder ball shear strength. In general, faster ram speed can result in higher ball shear strength.

Therefore, the characterization of solder ball shear strength is loading rate-dependent.

The Research Grant Council of Hong Kong sponsored this study through the grant of

HKUST6231/01E to the Hong Kong Univers ity of Science and

Technology (HKUST). The authors wish to acknowledge this support

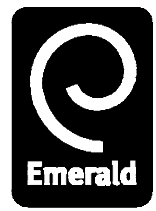

Soldering \& Surface Mount

Technology

14/ 1 [2002] 45-48

(C) MCB UP Limited

[ISSN 0954-0911]

DOI 10.1108/09540910210416468

\section{Introduction}

In the past decade, BGA technologies have overwhelmed the whole IC packaging industry. This is because BGA packages have many advantages over conventional ones. Among them are larger number of I/Os, self-alignment capability, more robust assembly process and better thermal/electrical performance (Lau, 1996; 2000; Lau and Pao, 1997). Furthermore, BGA packages are compatible with surface mount technology (SMT), consequently, leading to high throughput and low cost assemblies for mass production. For surface mount components (SMCs), the solder joints are not only the passage of electrical signals, power and ground, but also act as the mechanical support to hold the module in position on the printed circuit board (PCB). Therefore, solder joint reliability is a major concern for BGA packages.

Currently, the most popular method to evaluate the strength of solder ball attachment is the ball shear test. The typical value of solder ball shear strength for PBGA packages may be around $1000 g_{\mathrm{f}}$ (for 30-mil solder balls) (Coyle et al., 1999; Lee et al., 2000a; Levis and Mawer, 2000). Although such tests are simple and convenient to implement, there is not much mechanics justification to interpret the testing results. Most people still use the method of A-to-B comparison to determine the acceptance of packages. In July 2000, JEDEC published a new standard, JESD22-B117, for BGA solder ball shear tests (JEDEC, 2000). In this publication, the testing condition is specified in terms of the shear ram height only. The specification is that the gap between the shear ram tip and the ball mounting surface should be larger than $0.05 \mathrm{~mm}$ ( 2 mils) and smaller than (or equal to) 25 per cent of the ball height. However, another important testing parameter, namely, the shear ram speed (loading rate), is not addressed in this standard. The lack of specification in the shear speed may cause confusion for the comparison of solder ball shear strength characterized with different loading rates (Coyle and Solan, 2000; Hasegawa et al., 2000). Therefore, further research effort is required for understanding the rationale of solder ball shear tests and for investigating the effect of shear speed.

The present study is aimed at establishing a finite element model for the analysis of ball shear tests in order to evaluate the solder ball attachment strength of PBGA packages. In particular, the emphasis is placed on investigating the effect of the shear speed. The results obtained from this computational modeling analysis will be validated by experimental data.

\section{Solder ball shear tests}

The purpose of solder ball shear tests in the present study was for model validation. Solder balls under evaluation were standard $0.76 \mathrm{~mm}$ (30 mils) $63 \mathrm{Sn}-37 \mathrm{~Pb}$ spheres for PBGA packages. The substrates for ball attachment were BT laminates with a thickness of $0.46 \mathrm{~mm}$. The solder bond pads were solder-mask-defined with an opening of $0.6 \mathrm{~mm}$ in diameter. The metallization of bond pads was $\mathrm{Cu}$ with Ni/Au electro-plating. The solder balls were attached to the bond pads using a standard SMT reflow profile. After reflow, the average ball height and diameter were $0.64 \mathrm{~mm}$ and $0.78 \mathrm{~mm}$, respectively. The ball shear tests were conducted using a Dage-4000S machine. In the present study, the shear ram height was fixed at $10 \%$ of the solder ball height (i.e. $64 \mu \mathrm{m}$ ), which complies with the new JEDEC standard, but the shear ram speed varied $(20 \mu \mathrm{m} / \mathrm{s}, 50 \mu \mathrm{m} / \mathrm{s}, 100 \mu \mathrm{m} / \mathrm{s}$, $200 \mu \mathrm{m} / \mathrm{s}$ and $500 \mu \mathrm{m} / \mathrm{s}$ ). For each testing condition, 25 solder balls were sheared. The peak shear forces were recorded and the calculated average was taken as the ball shear strength. The testing results of ball shear strength are presented in Table I.

\section{Finite element model}

In the present study, both 2-D and 3-D finite element models were built and used in the analyses. The geometric models were based on the average dimensions measured from the cross-section of specimens for solder ball shear tests. A commercial finite element code, ANSYS Release 5.6, was used to perform the computational analysis. Figure 1 shows the 2-D model simulating the ball shear test. The finite element meshes consisted of the solder ball, the $\mathrm{Cu}$ bond pad, and the BT substrate, which were modeled by 8-node plane strain elements (PLANE183). The shear ram was considered a rigid body. A feature in ANSYS using the surface-to-surface target element (TARGE169) and the contact element (CONTA172) was employed to simulate the contact between the shear ram and the solder ball. The left right, and bottom sides of BT substrate were fixed as rigid boundaries. In the present 2-D model, there were 1582 nodes, 490 PLANE1 83 elements, 8 TARGE169 elements and 40 CONTA172 elements. Since this was a timedependent non-linear analysis, both large deformation and transient options were enabled in the analysis.

For the three-dimensional analysis, 20-node solid elements (SOLID186) were used to mesh the 3-D model. Due to the symmetry, only one half of the solder ball was modeled as shown in Figure 2. Similar to the 2-D modeling above, the surface-to-surface target element (TARGE170) and contact element (CONTA174) were employed to simulate the contact between the shear ram and the solder ball during the solder ball shear test. In the 3-D model, there were 11277 nodes, 2520 SOLID186 elements, 249 TARGE170 elements and 240 CONTA174 elements. The material properties and other modeling features for the 3-D model were the same as those assumed in the 2-D analysis.

\section{Constitutive models for the materials}

In the present computational model, all materials, except the solder ball, are assumed to behave as linear elastic materials. The material properties used in modeling are listed in 
S.W. Ricky Lee and Xingjia Huang Analysis on solder ball shear testing conditions with a simple computational model

Soldering \& Surface Mount

Technology

14/ 1 [2002] 45-48

Table

Ball shear strength under various shear speed

\begin{tabular}{|c|c|c|c|c|c|}
\hline Fixed ram height $(64 \hat{m})$ & 20 & 50 & $\begin{array}{c}\text { ar speed }(\hat{1} \\
100\end{array}$ & 200 & 500 \\
\hline $\begin{array}{l}\text { Testing } \\
\text { Modeling }\end{array}$ & $\begin{array}{c}1131(4 \%) \\
1143\end{array}$ & $\begin{array}{c}1171(5 \%) \\
1202\end{array}$ & $\begin{array}{c}1244(6 \%) \\
1244\end{array}$ & $\begin{array}{c}1350(4 \%) \\
1291\end{array}$ & $\begin{array}{c}1418(3 \% \\
1350\end{array}$ \\
\hline
\end{tabular}

Figure 1

2-D finite element model

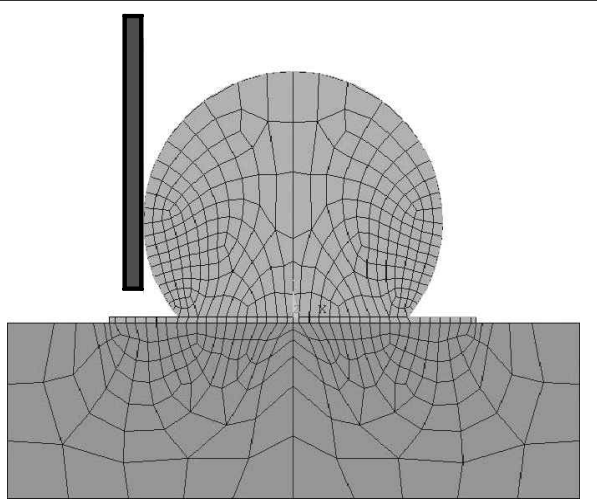

Table II. In the present study, the solder is considered as a visco-plastic material, which follows the Garofalo-Arrhenius creep constitutive (Lau, 1995):

$$
\frac{\mathrm{d} \gamma}{\mathrm{d} t}=C\left(\frac{G}{\Theta}\right)\left[\sinh \left(\omega \frac{\tau}{G}\right)\right]^{n} \exp \left(-\frac{Q}{k \Theta}\right)
$$

where $\gamma$ is the steady-state creep shear strain; $\mathrm{d} \gamma / \mathrm{d} t$ is the steady-state creep shear strain rate; $t$ is the time; $C$ is a material constant; $G$ is the temperature-dependent shear modulus; $\Theta$ is the absolute temperature $\left({ }^{\circ} \mathrm{K}\right) ; \omega$ defines the stress level at which the power law stress dependence breaks down; $\tau$ is the shear stress, $n$ is the stress exponent; $Q$ is the activation energy for diffusion mechanism; and $k$ is the Boltzmann constant $\left(8.617 \times 10^{-5} \mathrm{eV} /{ }^{\circ} \mathrm{K}\right)$, Darveaux and Banerji modified equation (1) to

$$
\frac{\mathrm{d} \gamma}{\mathrm{d} t}=A\left[\sinh \left(\frac{\tau}{B}\right)\right]^{n} \exp \left(-\frac{Q}{k T}\right)
$$

and have characterized the coefficients, $A, B, n$, and $Q$, for a few solder materials (Darveaux and Banerji, 1992). In

\section{Figure 2}

3-D finite element mode

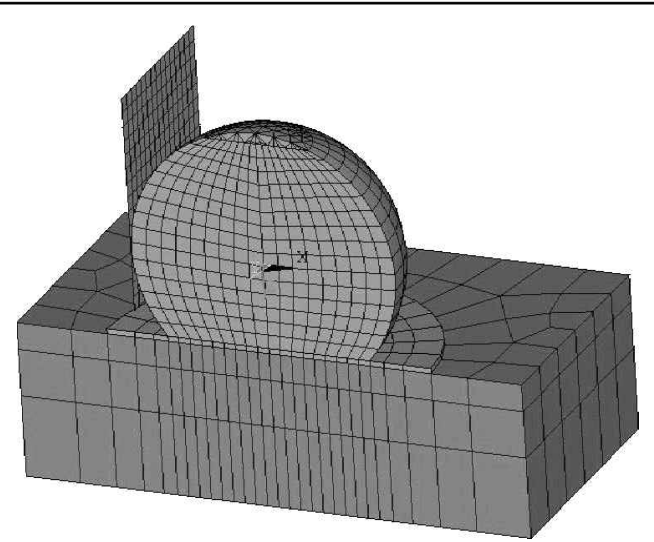

ANSYS, equation (2) is called the implicit creep model and the equation is further modified as:

$$
\frac{\mathrm{d} \varepsilon}{\mathrm{d} t}=C_{1}\left[\sinh \left(C_{2} \sigma\right)\right]^{C_{3}} \exp \left(-\frac{C_{4}}{T}\right)
$$

where $\sigma$ is the effective stress and $\mathrm{d} \varepsilon / \mathrm{d} t$ is the steady-state effective creep strain rate. The values of $C_{1}, C_{2}, C_{3}$, and $C_{4}$ in equation (3) for the eutectic solder are given in Table III (Lee et al., 2000b).

\section{Determination of effective thickness}

The direct result from the present computational analysis is the force-displacement curve simulating the ball shear test. It should be noted that, for the 2-D plane strain analysis, the force output from ANSYS was in the unit of N/mm (force per unit thickness). However, the actual diameter of solder was $0.78 \mathrm{~mm}(<1 \mathrm{~mm})$. In order to obtain the meaningful shear force for comparison, an effective thickness (scale factor) should be defined. Based on the fact that the ball mounting pad has a diameter of $0.6 \mathrm{~mm}$ and the solder bal has an axisymmetric geometry, it may be concluded that the effective thickness of the 2-D model must be smaller than $0.6 \mathrm{~mm}$. With such an observation, the scale factor for the effective thickness could be estimated as follows:

In the present study, the shear test condition with $64 \mu \mathrm{m}$ ram height and $100 \mu \mathrm{m} / \mathrm{s}$ shear speed was chosen as the base case. For this base case, the peak shear force obtained from ANSYS was $2969 \mathrm{~g}_{\mathrm{f}} / \mathrm{mm}$. Compared with the corresponding experimental data $\left(1244 g_{\mathrm{f}}\right.$, see Table I), the effective thickness, $t$, can be determined as:

$$
t=\frac{1244 g_{\mathrm{f}}}{2969 g_{\mathrm{f}} / \mathrm{mm}}=0.42 \mathrm{~mm}
$$

Figure 3 shows the original shear force-displacement curve from ANSYS and the modified curve with an effective thickness of $0.42 \mathrm{~mm}$ for the base case. Subsequently, the same value of effective thickness was applied to all other 2-D analyses. If the same effective thickness could yield to good agreement between testing and modeling for all cases (which will be shown in the next section), then it is reasonable to conclude that the selected effective thickness

\section{Table II}

Elastic material properties for modeling

\begin{tabular}{lrcc}
\hline Materials & $\boldsymbol{E}(\mathbf{M P a})$ & $\backslash$ & $\nabla\left(\mathbf{g} / \mathbf{c m}^{\mathbf{3}}\right)$ \\
\hline 63Sn-37Pb & 29,800 & 0.40 & 8.41 \\
Cu pad & 128,700 & 0.34 & 8.31 \\
BT Substrate & 14,000 & 0.39 & 1.2 \\
\hline
\end{tabular}

Table III

Creep option input parameters for ANSYS

\begin{tabular}{llll}
\hline $\boldsymbol{C}_{\mathbf{1}}\left(\mathbf{s}^{-1}\right)$ & $\boldsymbol{C}_{\mathbf{2}}\left(\mathrm{MPa}^{-1}\right)$ & $\boldsymbol{C}_{\mathbf{3}}$ & $\boldsymbol{C}_{\mathbf{4}}\left({ }^{\circ} \mathbf{K}\right)$ \\
\hline $\mathbf{3 3 9 . 0 1 0 2}$ & 0.062653 & 3.3 & 6,360 \\
\hline
\end{tabular}


S.W. Ricky Lee and Xingjia Huang Analysis on solder ball shear testing conditions with a simple computational model

Soldering \& Surface Mount Technology

14/ 1 [2002] 45-48

\section{Figure 3}

Modification of force-displacement curve

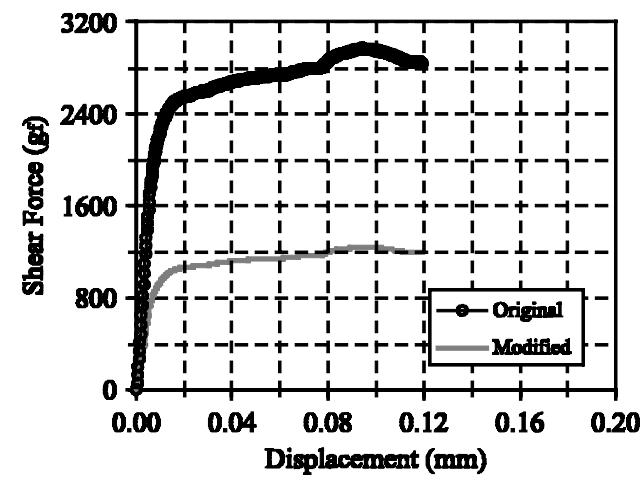

was not a random coincidence. It should carry a certain physical meaning, which represents the scale factor between the 2-D and 3-D analyses. Furthermore, the fact that the chosen effective thickness is smaller than $0.6 \mathrm{~mm}$ also enhances the confidence in the selected value.

\section{Comparison between 2-D and 3-D analyses}

The force-displacement curve obtained from the 3-D analysis for the base case is presented in Figure 4 and compared to the corresponding results from the 2-D analysis and experimental data. It is observed that the 2-D modeling fits the experimental data better than the 3-D analysis.

In general, it is considered that the 3-D model should give more accurate results than the 2-D analysis. However due to the accumulation of numerical truncation errors, this may not be always true for the large scale computation in the non-linear time-dependent analyses. In the present study, the degrees of freedom for the 2-D and the 3-D analyses were $3164(1582$ nodes $\times 2)$ and $33831(11277$ nodes $\times 3)$, respectively. With one order of magnitude in the number of degrees of freedom, the accumulation of numerical errors might play a significant role making the $3-\mathrm{D}$ analysis a worse case.

In addition, the 3-D analysis is usually very time consuming. For the comparison shown in Figure 4, while the 3 -D analysis took more than 3 days, the 2-D model only required $3 \mathrm{~h}$ using the same computing facility. Therefore, the 2-D analysis should be a better choice for the present study, as long as the proposed effective thickness model is validated.

Figure 4

Comparison of force-displacement curves

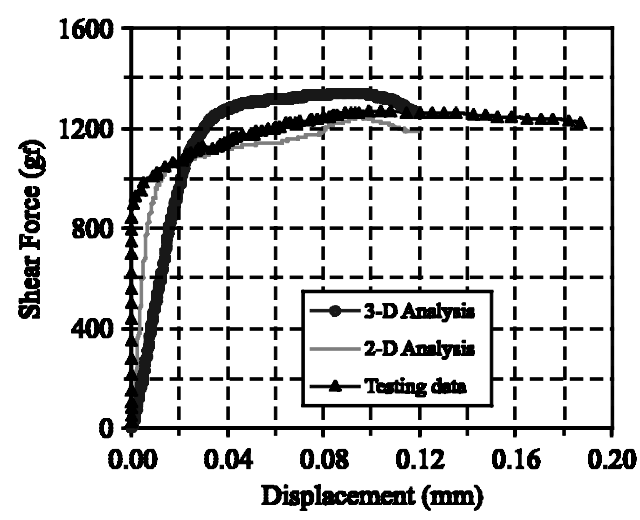

\section{Model validation and discussion}

Figures 5-8 present the results of 2-D analyses for the shear speeds of $20,50,200$ and $500 \mu \mathrm{m} / \mathrm{s}$, respectively. All corresponding experimental data are plotted as well for comparison. The peak shear forces, which are regarded as the ball shear strength, are also summarized in Table I. It is observed that the testing and modeling results are in good agreement for all cases. Since the scale factor was determined using the base case only, it is reasonable to conclude that the selected effective thickness was not a random coincidence. It should carry a certain physical meaning, which represents the scale factor between the 2-D

Figure 5

Force displacement curves for $204 \mu \mathrm{m} / \mathrm{s}$

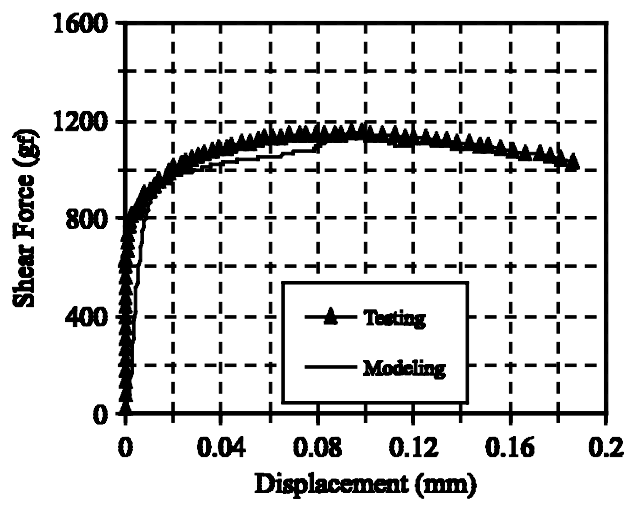

Figure 6

Force displacement curves for $50 \mu \mathrm{m} / \mathrm{s}$

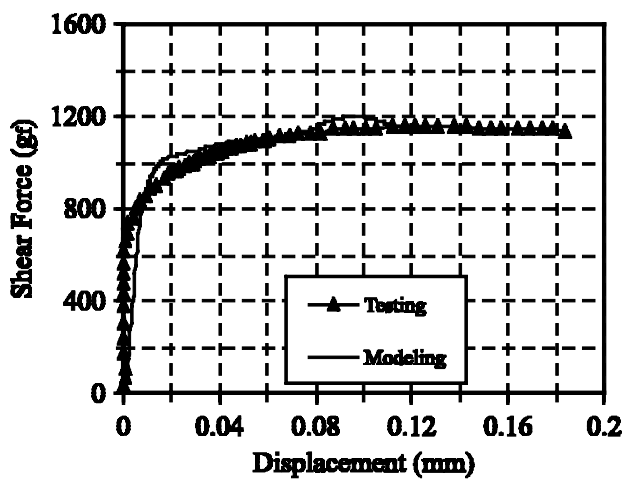

Figure 7

Force displacement curves for $200 \mu \mathrm{m} / \mathrm{s}$

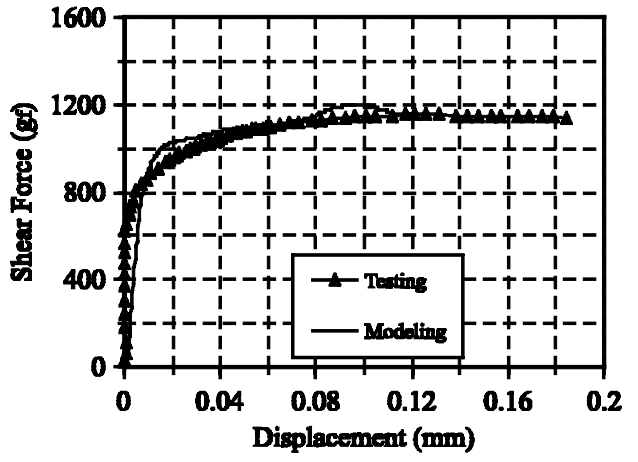


S.W. Ricky Lee and Xingjia Huang Analysis on solder ball shear testing conditions with a simple computational model

Soldering \& Surface Mount Technology

14/ 1 [2002] 45-48
Figure 8

Force displacement curves for $500 \mu \mathrm{m} / \mathrm{s}$

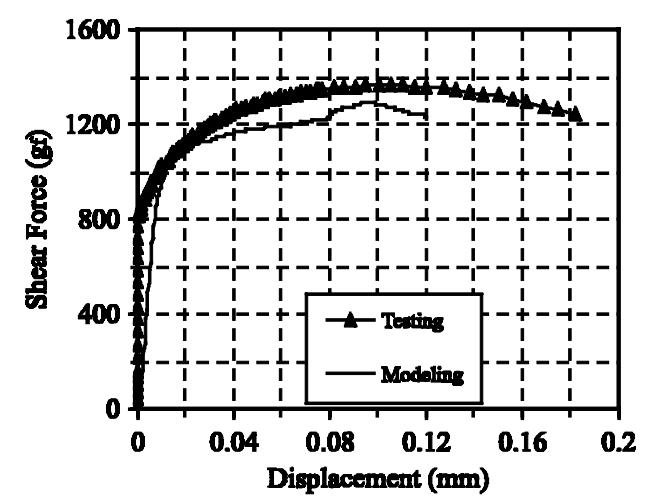

and 3-D analyses. Based on the good agreement in comparison, the concept of effective thickness is validated.

For the ease of summarizing the general trends of ball shear strength, the data given in Table I are plotted in Figure 9. From this figure, one can find that, the ball shear strength increases with the increase of shear speed. Anothe point to be noted is that, although in general the shear strengths from testing and modeling are in good agreement, there is a notable discrepancy for the cases with shear speed faster than $100 \mu \mathrm{m} / \mathrm{s}$. From this point of view, it may be deduced that the ideal solder ball shear testing conditions are the cases with shear speed slower than $200 \mu \mathrm{m} / \mathrm{s}$.

\section{Summary and conclusions}

In the present study, both 2-D and 3-D finite element models were developed for the analysis of solder ball shear tests. The effect of shear speed on solder ball shear strength was studied not only by experimental investigation, but also by computational modeling. The results are summarized as follows.

- The shear speed has substantial effects on the solder bal shear strength. The data from both testing and modeling indicates that faster shear speed would results in higher ball shear strength.

\section{$\overline{\text { Figure } 9}$}

Effect of shear speed on the ball shear strength

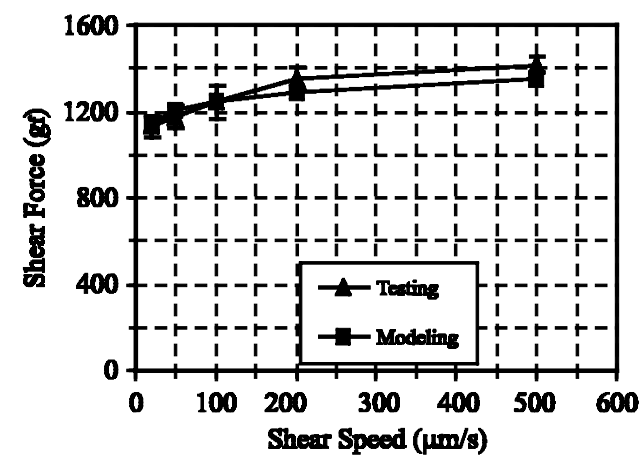

- The results from testing and 2-D modeling were in good agreement. An effective thickness was identified for the 2-D plane strain analysis. With such a scale factor, it is feasible to study 3 -D problem with a 2-D finite element model.

- The ideal solder ball shear test conditions were recommended to be the cases with shear speed slower than $200 \mu \mathrm{m} / \mathrm{s}$.

It should be noted that although the present analyses were performed for solder balls of BGA package with $30 \mathrm{mi}$ solder balls, the methodology may be applied to other cases such as BGA packages with smaller solder balls and the solder bumps of flip chips. With the assistance of computational modeling, it seems that it is possible to forecast the ball shear strength under various testing conditions as long as a base case is tested in advance. In addition, with further stress analyses, it may be possible to achieve more meaningful comparisons among different packages. The results obtained from this study should be very helpful for the electronics manufacturing industry to interpret their testing data and determine the acceptance criteria for the products with solder ball attachment.

\section{References}

Coyle, R.J. and Solan, P.P. (2000), "The influence of test parameter and package design features on ball shear test requirements", 26th IEEE/CPMT International Electronics Manufacturing Technology Symposium, pp. 168-77.

Coyle, R.J., Holiday, A., Mescher, P., Solan, P.P., Gahr, S.A., Cyker, H.A., Dorey, J.K. and Ejim, T.I. (1999), "The influence of nickel/gold surface finish on the assembly quality and long term reliability of thermally enhanced BGA packages", 24th IEEE/CPMT International Electronics Manufacturing Technology Symposium, pp. 23-35.

Darveaux, R. and Banerji, K. (1992), "Constitutive relations for tinbased solder joints", Proceedings of IEEE Electronic Components \& Technology Conference, pp. 538-51.

Hasegawa, K., Takahashi, A., Noudou, T., Nakajima, S., Takahashi, A., Nomoto, M. and Nakaso, A. (2000), "Electroless Ni$\mathrm{P} / \mathrm{Pd} / \mathrm{Au}$ plating for semiconductor package substrate", SMTA International Proceedings of the Technical Program, Edina, $\mathrm{MN}$, pp. 225-31.

JEDEC (2000), "BGA Ball Shear", JEDEC Solid State Technology Association JESD22-B117, July.

Lau, J.H. (1995), Ball Grid Technology, McGraw-Hill, New York, NY

Lau, J.H. (1996), Flip Chip Technologies, McGraw-Hill, New York, NY.

Lau, J.H. (2000), Low Cost Flip Chip Technologies, McGraw-Hill, New York, NY.

Lau, J.H. and Pao, Y.H. (1997), Solder Joint Reliability of BGA, CSP, Flip Chip, and Fine Pitch SMT Assemblies, McGraw-Hill, New York, NY.

Lee, S-W.R., Newman, K. and Hu, L. (2000b), “Thermal fatigue analysis of PBGA solder joints with the consideration of damage evolution", Packaging of Electronic and Photonic Devices, EEP-Vol. 28, November 5-10, FL, pp. 207-12.

Lee, S-W.R., Yan, C.C., Karim, Z. and Huang, X. (2000a), "Assessment on the effect of Electroless nickel plating on the reliability of solder ball attachment to the bond pads of PBGA substrate", Proc. 50th ECTC, May 21-24, NV, pp. 868-73.

Levis, K.M. and Mawer, A. (2000), "Assembly and solder joint reliability of plastic ball grid array with lead-free versus lead-tin interconnect", Proc. 50th ECTC, May 21-24, NV, pp. 1198-204. 